\title{
A Turbidity Test Based Centrifugal Microfluidics Diagnostic System for Simultaneous Detection of HBV, HCV, and CMV
}

\author{
Hung-Cheng Chang, ${ }^{1}$ Yu-Tin Chao, ${ }^{1}$ Jia-Yush Yen, ${ }^{1}$ Ya-Lin Yu, ${ }^{1}$ Chun-Nan Lee, ${ }^{2}$ \\ Bing-Ching Ho, ${ }^{2}$ Kou-Chen Liu, ${ }^{3}$ Jiunn Fang, ${ }^{4}$ Chii-Wann Lin, ${ }^{5}$ and Jiun-Haw Lee ${ }^{6}$ \\ ${ }^{1}$ Department of Mechanical Engineering, National Taiwan University, Taipei 106, Taiwan \\ ${ }^{2}$ College of Medicine, National Taiwan University, Taipei 106, Taiwan \\ ${ }^{3}$ Department of Electronic Engineering, Chang Gung University, Taoyuan 333, Taiwan \\ ${ }^{4}$ Department of Aerospace and System Engineering, Feng Chia University, Taichung 407, Taiwan \\ ${ }^{5}$ Institute of Biomedical Engineering, National Taiwan University, Taipei 106, Taiwan \\ ${ }^{6}$ Department of Electronic Engineering, National Taiwan University, Taipei 106, Taiwan
}

Correspondence should be addressed to Jia-Yush Yen; jyen@ntu.edu.tw

Received 2 October 2014; Accepted 24 November 2014

Academic Editor: Timo Sajavaara

Copyright ( 92015 Hung-Cheng Chang et al. This is an open access article distributed under the Creative Commons Attribution License, which permits unrestricted use, distribution, and reproduction in any medium, provided the original work is properly cited.

\begin{abstract}
This paper presents a LAMP- (loop-mediated isothermal amplification-) based lab-on-disk optical system that allows the simultaneous detection of hepatitis B virus, hepatitis C virus, and cytomegalovirus. The various flow stages are controlled in the proposed system using different balance among centrifugal pumping, Coriolis pumping, and the capillary force. We have implemented a servo system for positioning and speed control for the heating and centrifugal pumping. We have also successfully employed a polymer light-emitting diode section for turbidity detection. The easy-to-use one-click system can perform diagnostics in less than 1 hour.
\end{abstract}

\section{Introduction}

Centrifugal microfluidics offers convenient pumping and valving mechanisms and can easily be configured to use disposable disks for complete sample-to-answer designs. These features have made centrifugal microfluidics devices very popular for genotypic analysis systems, and many innovative designs have been developed based on this principle.

This paper presents a loop-mediated isothermal amplification- (LAMP-) based lab-on-disk system designed to allow the simultaneous detection of hepatitis B virus (HBV), hepatitis $\mathrm{C}$ virus (HCV), and cytomegalovirus (CMV). LAMP is basically a single-tube technique for the amplification of DNA. LAMP uses three pairs of primers to recognize sections of the target sequence. LAMP produces more DNA than does the traditional polymerase chain reaction (PCR) technique and it is less sensitive to PCR inhibitors in the blood, which has led to it being used to detect many infectious diseases, including mycobacterium tuberculosis [1-4], severe acute respiratory syndrome [5], human influenza [6-8], avian influenza [9], herpes $[10,11]$, malaria $[12,13]$, and papillomavirus [14].

HBV is detected in blood tests for viral antigens and antibodies, but the window periods of these tests vary. Molecular detection of HBV is therefore very important. Because $\mathrm{HCV}$ is very difficult to culture, its detection mainly depends on molecular diagnosis $[13,15]$. An antigen assay was recently developed for the early detection of $\mathrm{HCV}$ infection. $\mathrm{HCV}$ is classified into six genotypes, among which the RNA genotypes vary by $30 \sim 35 \%$, which makes it necessary to adopt a conservative sequencing technique [16]. There are several antibody tests available for CMV, as well as qualitative and quantitative PCR tests. Patients with CMV infection are often not tested since the condition induces very few symptoms, and so a convenient test procedure would be very desirable [17]. 
Centrifugal microfluidics has been widely studied as a convenient and inexpensive testing mechanism. It is easy to add valving, volume metering, mixing, and flow switching function to the inherent centrifugal pumping in order to satisfy the requirements in a particular application. While many studies have demonstrated the required biological analysis steps $[18,19]$, not many results are available for sampleto-answer systems using centrifugal microfluidics [20-22]. Schembri et al. reported a disk analyzer that processes whole blood using 12 different tests [20]. Although that system is very capable, it uses very complex specially made disposable disks. The Madou compact disk (CD) platform balances the centrifugal force and the capillary force in order to integrate functions such as flow sequencing, micromixing, and capillary metering onto a CD [21]. Reference [22] designed a very complicated flow pattern to implement an automated enzyme-linked immunosorbent assay (ELISA) [22]. They used laser-irradiated ferrowax microvalves to control the procedures in microbead-based suspension ELISAs, including plasma separation, incubation, washing, enzyme reaction, and absorbance detection.

The LAMP procedure in our microfluidic lab-on-disk system is a high-sensitivity and high-specificity one-step DNA amplification process. Recently there have been several reports on the successful use of LAMP to detect both HBV and $\mathrm{HCV}[16,23-26]$. Moreover, the desire for a convenient CMV test illustrates the need for multipurpose diagnostic procedures. LAMP uses a one-step incubation for parallel testing, which prompted us to look into simultaneously combining the tests for all three viruses on a single disk.

One important advantage of LAMP is the easy reading based on turbidity. The detection section on the test disk in our design was implemented by building a bright polymer light-emitting diode (PLED) at the bottom of the detection reservoir (Figure 3) for providing illumination when LAMP is completed. The turbidity of the test sample can then be easily read by the naked eye or by the standard AOI technique.

The proposed overall system employs a novel centrifugal microfluidic design that uses a combination of centrifugal pumping and Coriolis force for flow control. The inclusion of a carefully designed valving system achieves very effective parallel staging of the flow for the LAMP incubation and PLED detection.

\section{Materials and Methods}

2.1. Research Methods. Liquids on a disk are easily moved from the center to the outer perimeter by the centrifugal force that creates an artificial gravity in the rotating-disk reference frame. Flow control on rotating disks can be achieved by a capillary valve that relies on the capillary force to stop the flow in microchannels. Passive capillary-burst valves are hydrophobic patches that block a flow until a specific rotational speed is reached [27]. Their principle of operation is based on a pressure barrier that develops when the cross section of the capillary expands abruptly. Another essential feature of the microfluidic system is that the Coriolis pseudoforce can prevail over all other forces beyond a certain

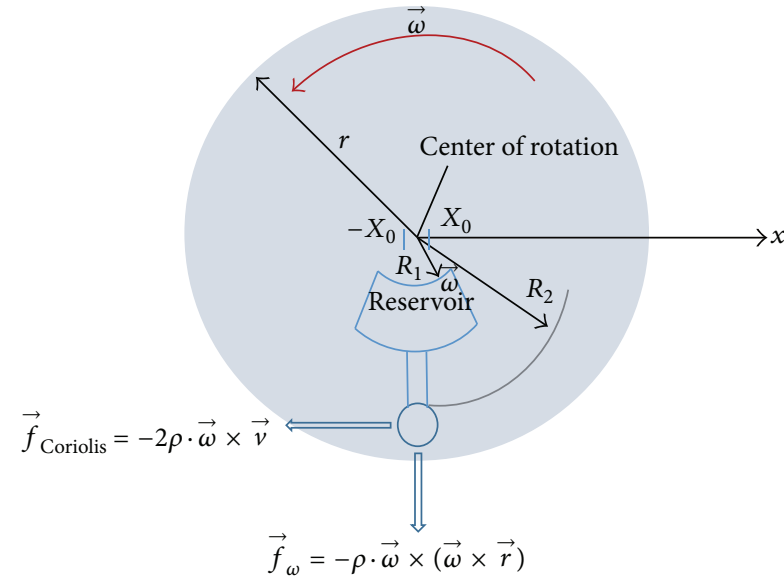

FIgURE 1: The width of the channel is $\Delta x=2 x_{0}$, and $R_{1}$ and $R_{2}$ are the boundaries of a liquid element corresponding to its distances from the center of the $\mathrm{CD}$. When $\mathrm{CD}$ spinning at induces the radial centrifugal force and transversal Coriolis force with respect to the flow velocity and the mass density of the liquid $\rho$.

speed of rotation. A sufficiently strong Coriolis force can divert the fluid flow based on the direction of rotation.

A capillary barrier can be regarded as a valve with respect to rotation speed $\omega$. There are three forces that can be described (Figure 1) [28]. The continuum of incompressible fluids is given by the Navier-Stokes equation

$$
\rho\left[\frac{\partial}{\partial t} \vec{v}+(\vec{v} \cdot \nabla) \vec{v}\right]=-\nabla p+\eta \nabla^{2} \vec{v}
$$

and the Coriolis force term is

$$
\vec{f}_{\text {Coriolis }}=-2 \rho \cdot \vec{\omega} \times \vec{v},
$$

where $\rho$ is the mass density of the liquid and $r$ is the distance of a liquid element from the center of the CD [27]. Consider a liquid plug on the disk defined by its inner and outer radial positions $\left(R_{1}\right.$ and $R_{2}$, resp.), a radial length $l=R_{2}-R_{1}$, and

$$
\bar{R}=\frac{1}{2}\left(R_{1}+R_{2}\right)
$$

as its mean radial position.

Assuming that there is flow through a gap equal to the width between the walls located at $x \pm x_{0}$,

$$
\Delta x=2 x_{0} .
$$

In the steady state $d w / d t=0$ and no rotational Euler acceleration needs to be considered. In this condition the Coriolis force can overcome the centrifugal force at angular velocities higher than a certain threshold.

Consider the effects of the capillary force.

The ratio between $\vec{f}_{w}$ and $\vec{f}_{\text {Coriolis }}$ becomes

$$
\frac{\left|\vec{f}_{\omega}\right|}{\left|\vec{f}_{\text {Coriolis }}\right|}=\frac{\rho^{2} \omega^{3} \bar{R} l \Delta x^{3}}{16 \eta \sigma \cos \theta}
$$




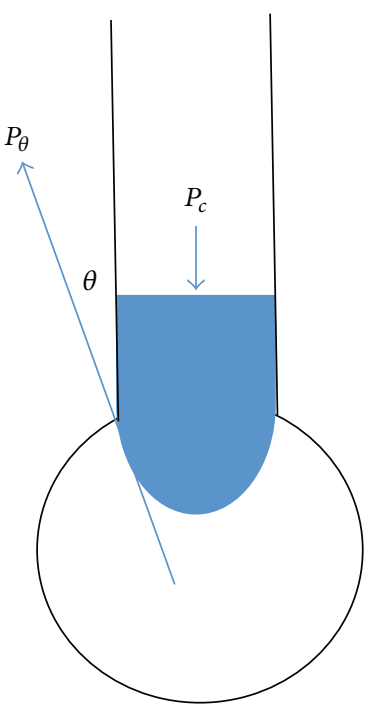

FIGURE 2: Illustration of valve interaction.

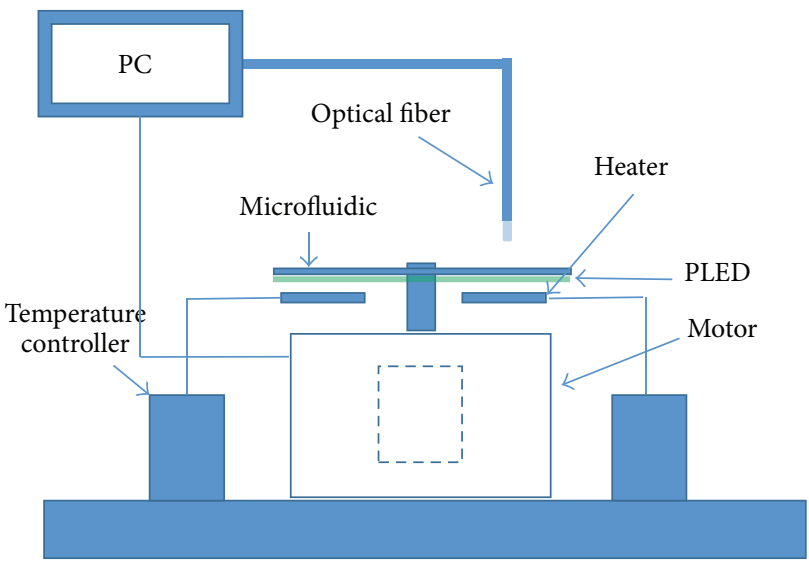

FIGURE 3: Experimental setup for CD microfluidic testing.

which is highly dependent on $\omega$ and $x_{0}$. This indicates that surface tension has a minor impact since it only becomes significant when the rotation speed is far beyond the critical threshold.

In Figure 2, $P_{c}$ is the pumping force per unit area and $P_{s}$ is the capillary force per unit area. When liquid pressure $\Delta P_{c}$ prevails over capillary pressure $P_{\theta}$, the liquid will move from the channel into the reservoir according to

$$
\frac{d P_{c}}{d r}=\rho \omega^{2} r
$$

Thus, integrating (3) yields

$$
\Delta P_{c}=\rho \omega^{2} \cdot \Delta R \cdot \bar{R},
$$

where $\Delta R=R_{2}-R_{1}$. The capillary force per unit area $\left(P_{s}\right)$ equals the interfacial tension:

$$
\Delta P_{s}=P_{\theta}=\gamma \cdot l=\frac{4 \sigma \cos \theta}{\Delta x} .
$$

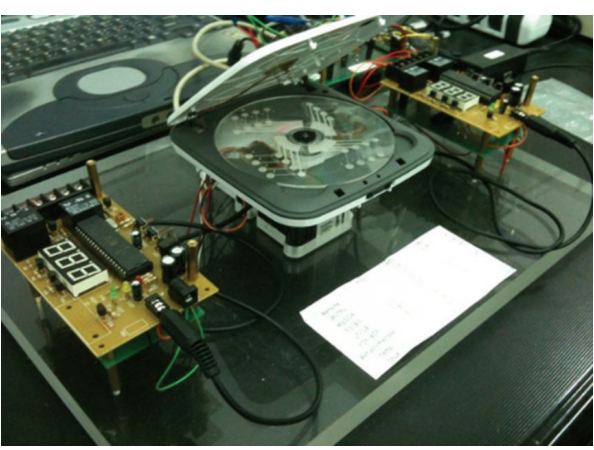

FIGURE 4: Photograph of the components of the biomedical diagnostic system.

The centrifugal force drives the sample liquid released from the reservoir when liquid pressure $\Delta P_{c}$ overcomes the capillary barrier $\left(P_{\theta}\right)$, and the liquid moves into the channel. The burst frequency is the rotational speed at which the fluids are released. Integrating (7) and (8) yields

$$
\omega_{\text {bust }}=\left(\frac{4 \sigma \cos \theta}{\Delta x \rho \Delta R \bar{R}}\right)^{1 / 2} .
$$

The burst frequency can be precisely controlled by appropriately choosing the dimensions and location of the channel.

2.2. Microfluidic Channels and System Design. The architecture of the diagnostic system, as shown in Figure 3, consists of four main components: the disposable microfluidic disk, the temperature control system, the servo motor and control unit, and the SPR detection system. The actual components are shown in Figure 4.

To enable simultaneous detection of different viruses, the test regions on the $\mathrm{CD}$ are directed into four radial channel paths: three for virus detection and the fourth for comparison. The system controls the flow staging by changing the balance between the centrifugal acceleration and the Coriolis acceleration. Such staged flow resistance divides the diagnostic process into discrete stages. The configuration of the microchannels is shown in Figure 5.

There are three test stages on the disk: (i) an input sample reservoir to house the original test sample and to aid even distribution of the sample into the four channels, (ii) a LAMP stage for virus incubation, and (iii) an illuminating stage for detection. The input stage uses centrifugal force to ensure that the liquid is distributed equally into the second stage. The second-stage reservoir is for the LAMP heating.

The first stage of the test is the input stage. We construct the first and second reservoirs with the same depth (the red region in Figure 5), while the third-stage reservoir (the yellow region) is considerably shallower. The fluid flows easily from the first reservoir into the second reservoir under the combined influence of the centrifugal force and the capillary force, and the second reservoir fills up with liquid from left to right. After the LAMP process is completed, the system rotates the disk in the reverse direction and at a much higher speed. The combined strong centrifugal pumping and the 


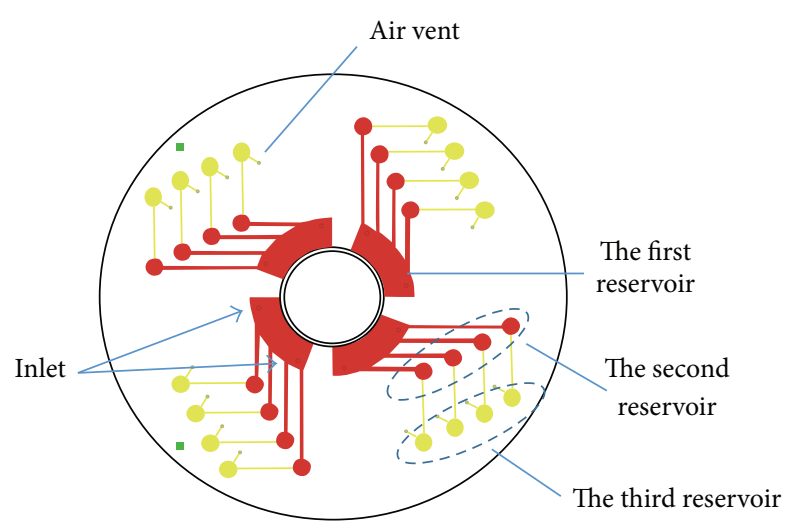

FIGURE 5: Configuration of the microfluidic channels.

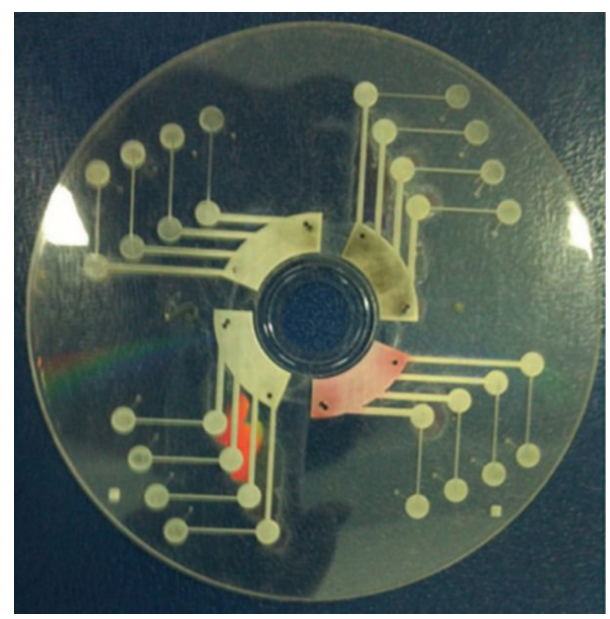

FIGURE 6: The microfluidic disk.

Coriolis force then drive the flow into the third stage from left to right for diagnostics. Figure 6 shows an actual CD with microfluidic channels.

The second stage is the LAMP stage. The depth difference between the second and third reservoirs stops the flow and restricts the fluid at the second stage for heating. The heating pads are capable of heating the samples to $65^{\circ} \mathrm{C}$ in 20 minutes. The control unit takes temperature measurements from an RTD-based temperature sensor and provides on-off control to the heating pads through two digital I/O terminals.

The depth of the curved channels could cause an evaporation problem during heating. This is solved by making the second reservoir much deeper than the next step along the flow route.

These mechanisms make it possible to control the reactant reacting at the reservoir, with LAMP occurring without dissipation due to heating and all reactants being controlled at their assigned reservoirs.

The third stage after the pumping requires lighting for the turbidity test. Our team developed the technology to deposit composite metal and oxide layers on the disk surface to form a fully transparent PLED and also to sputter on a $\mathrm{ZnO}$ layer for protective packaging [29] (Figure 7). The disk

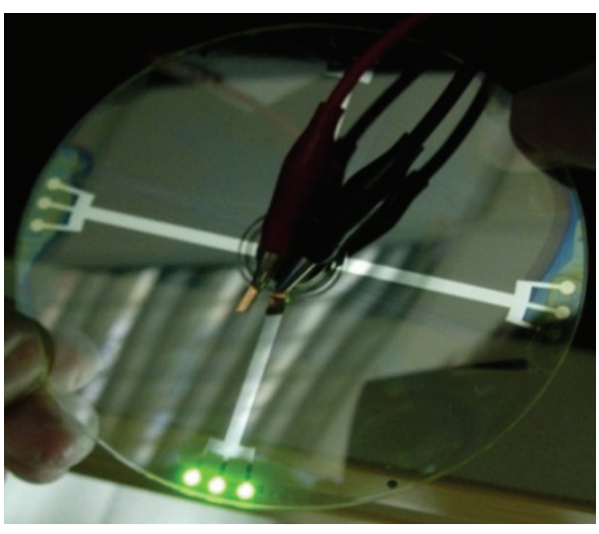

FIGURE 7: Illuminating PLED stage for turbidity examination.

surface contains conducting leads and the cover has electrical contacts for power input (Figure 8). After the final pumping action, the servo system will turn the disk to the turbidity test position and an automated procedure will light up the PLED and carry out the diagnostic procedure, with the results appearing on the user-interface monitor (Figure 9). The detection unit shines a laser on the detection stage for SPR detection. The occurrence of SPR at the corresponding angle provides an indication of whether the sample is infected.

\section{Results and Discussion}

3.1. Experiments on Fluid Flow in Microchannels. We performed experiments to investigate the two key points of pumping and blocking fluid flow in the microchannel. The simulation results suggested that capillary forces can be used to regulate flow in a rotating lab-on-disk system. Beyond a certain rotation-speed threshold, which is a function of the geometry of the channels, selecting the appropriate rotation speed and implementing a clockwise rotation can be used to stagnate the flow at the second reservoir (Figures 10(a)-10(b)).

Based on the capillary bursting occurring at certain angular velocities, as determined by the dimensions and location of the microchannel, according to (9), we were able to smoothly transport fluid to the second stage for LAMP. The rotation speed was initially $500 \mathrm{rev} / \mathrm{sec}$ and increased to $1500 \mathrm{rev} / \mathrm{sec}$ to drive the fluid filling in the second reservoir from left to right. The second processing step was based on using the Coriolis force to divert the fluid flow into the third stage from left to right in a counterclockwise direction at over $1600 \mathrm{rev} / \mathrm{sec}$. The results show that the centrifugal force can be used to propel the liquid and that combining the capillary force and Coriolis force allows perfect control of the fluids to the desired stage.

3.2. Optical Turbidity Test Based on LAMP Reaction. The LAMP reaction was performed according to the results of experiments and the test results as identified by turbidity. The amplification of DNA produces pyrophosphate, which exhibits white turbidity. The amplification was performed at $65^{\circ} \mathrm{C}$ in a laboratory for 1 hour, with the naked eye or a 


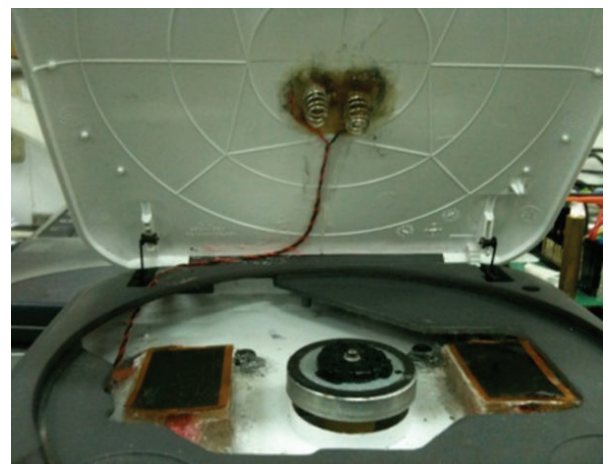

Figure 8: Electrical contacts to supply electricity to the test disk.

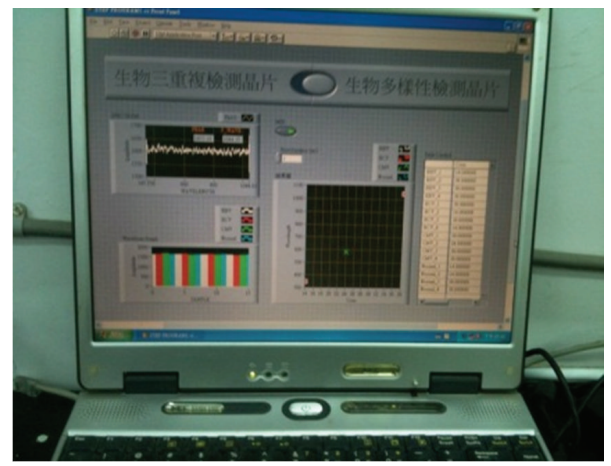

FIGURE 9: User interface for the automated control system.

TABLE 1: Reagent values for each virus.

\begin{tabular}{lcccc}
\hline & Unit & HBV & HCV & CMV \\
\hline Betaine & $\mathrm{M}$ & 1 & 0.8 & 0.8 \\
$\mathrm{MgSO}_{4}$ & $\mathrm{mM}$ & 4 & 6 & 10 \\
dNTPs & $\mathrm{mM}$ & 1.6 & 1.4 & 1.4 \\
Bst polymerase & $\mathrm{U}$ & & 8 & \\
F3/B3 & $\mu \mathrm{M}$ & & 0.2 & \\
$\mathrm{LF} / \mathrm{LB}$ & $\mu \mathrm{M}$ & 0.4 & 1 & 0.8 \\
FIP/BIP & $\mu \mathrm{M}$ & 1.6 & 2 & 1.6 \\
Temperature & ${ }^{\circ} \mathrm{C}$ & 63 & 65 & 65 \\
Time & Minutes & 45 & 60 & 60 \\
DNA volume & $\mu \mathrm{L}$ & & 5 & \\
Sensitivity & Copies & 50 & 60 & 10 \\
\hline
\end{tabular}

fiberoptic sensor used to detect the solution turbidity during LAMP, which was then confirmed by the LAMP detection system.

For the experiments we prepared the reagents according to the proportions listed in Table 1 . The reaction volume of $3 \mu \mathrm{L}$ was added to a small centrifuge tube $(0.5 \mathrm{~mL})$. When the LAMP reaction occurred, $1 \mu \mathrm{L}$ of the DNA sample was mixed with $24 \mu \mathrm{L}$ of the LAMP reagent, of which a $2.5 \mu \mathrm{L}$ aliquot was placed in the PCR machine. LAMP lasted for 45 minutes, and then electrophoretic analysis was performed. Five concentrations were used $(20 \mathrm{ng} / \mathrm{mL}, 2 \mathrm{ng} / \mathrm{mL}, 0.2 \mathrm{ng} / \mathrm{mL}, 20 \mathrm{pg} / \mathrm{mL}$, and $2 \mathrm{pg} / \mathrm{mL}$ ) (Figure 11) to confirm the analysis results for the reactant.

A $2.5 \mu \mathrm{L}$ aliquot of the liquid mixture was placed in the reservoir of the chip and covered with $1.5 \mu \mathrm{L}$ of mineral oil. An Al substrate was used for sealing together with the heating plate, and electrophoretic analysis was performed. We found that the signal intensity (RU) is obviously relative to $R^{2}=0.985$ (Figure 12). The use of different reaction intensities in an SPR LAMP detection system will yield different concentrations of the sample. Although it is difficult to observe changes in the luminance from Figure 2, it is easy to distinguish the reaction turbidity in Figure 3. The obtained results indicate that the SPR LAMP detection system-using a spectrometer to detect and examine the spectrum signal via a human interface-can be used to assess the degree of turbidity, making it possible to detect viruses on the CD.

\section{Conclusion}

This study designed a fully automated optical biomedical diagnostic LAMP-based lab-on-disk system. LAMP amplification can easily be carried out by measuring luminous intensity of DNA samples at minimal cost and using a disposable disk. To implement the SPR procedures, we deposited an organic light-emitting diode on the test disk with electricityconducting leads and designed the microchannel, by means of the Coriolis force, centrifugal force, and capillary force, to control sample flow into the reservoir. The samples are 


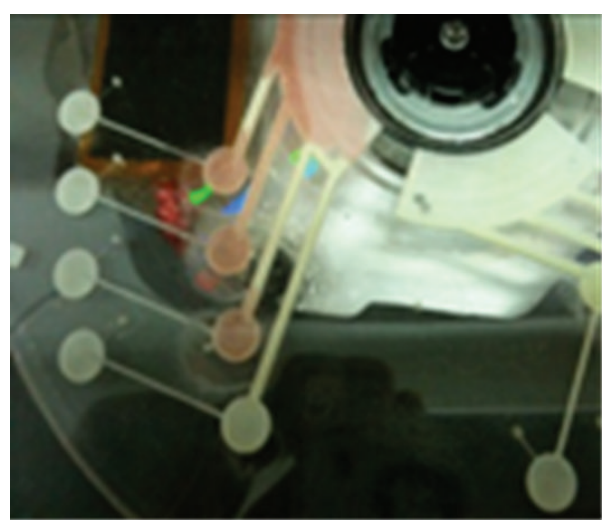

(a)

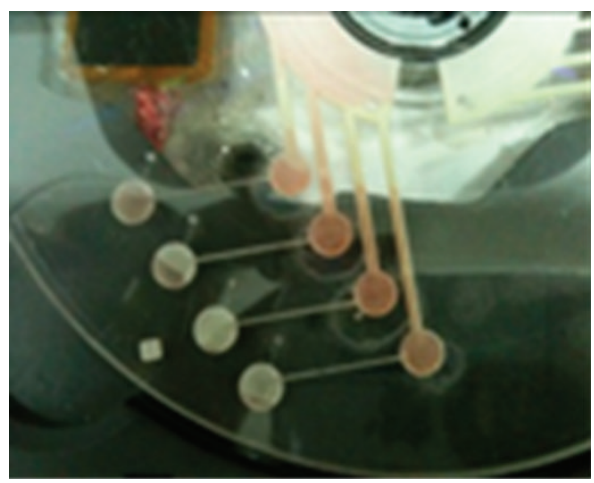

(c)

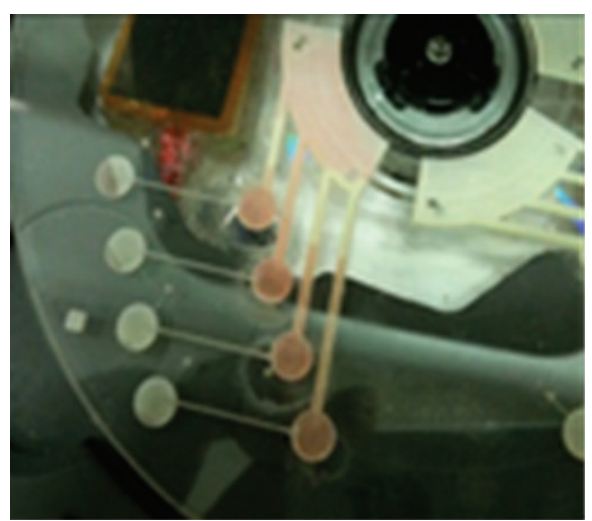

(b)

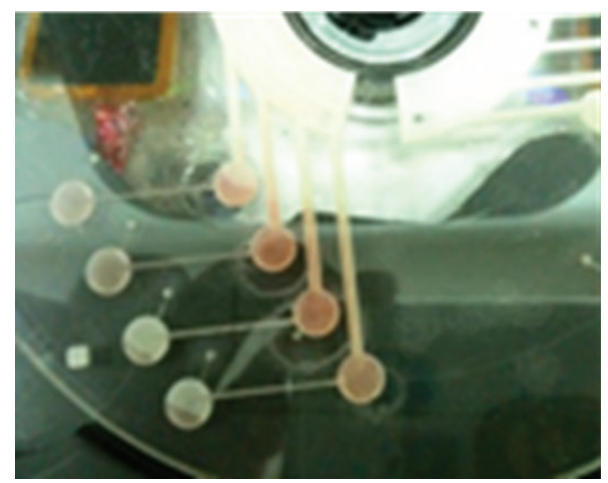

(d)

FIGURE 10: Fluid flows for rotation speeds of (a) 660, (b) 1500, (c) 1600, and (d) 1700 rev/sec. The rotation was in the clockwise direction for panels (a)-(b) and in the counterclockwise direction for panels (c) and (d).

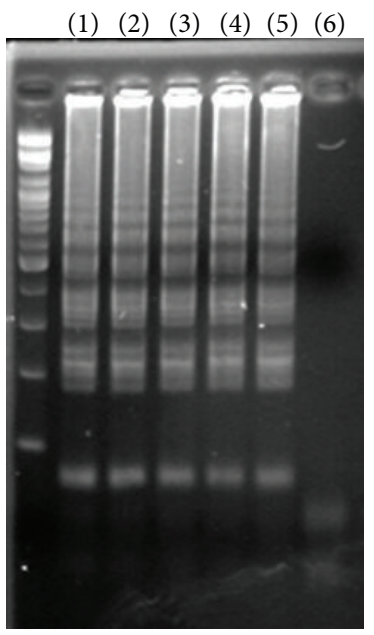

FIGURE 11: Turbidity in the traditional LAMP reaction for DNA samples at various concentrations: (1) $20 \mathrm{ng} / \mathrm{mL}$, (2) $2 \mathrm{ng} / \mathrm{mL}$, (3) $0.2 \mathrm{ng} / \mathrm{mL}$, (4) $20 \mathrm{pg} / \mathrm{mL}$, (5) $2 \mathrm{pg} / \mathrm{mL}$, and (6) negative control.

directed to sequentially flow into the system's four channels for the on-site detection of the three kinds of the virus: HBV (using 50 copies), HCV (using 60 copies), and CMV (using 10 copies). The key advantages of our system include

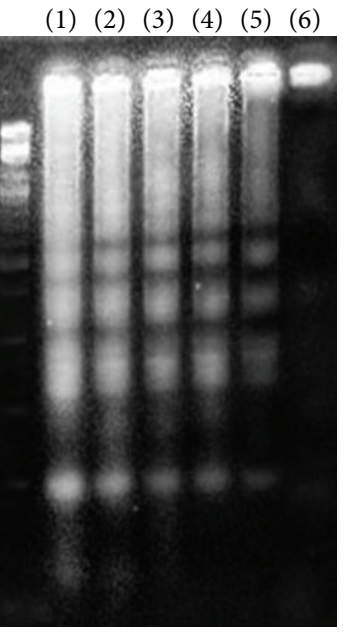

FIGURE 12: Turbidity in the SPR LAMP reaction in the reservoir of the chip for DNA samples at various concentrations: (1) $20 \mathrm{ng} / \mathrm{mL}$, (2) $2 \mathrm{ng} / \mathrm{mL}$, (3) $0.2 \mathrm{ng} / \mathrm{mL}$, (4) $20 \mathrm{pg} / \mathrm{mL}$, (5) $2 \mathrm{pg} / \mathrm{mL}$, and (6) negative control.

smaller sample quantity and decreased time needed for sample processing when compared to the requirements of a traditional LAMP reaction employing a PC-based glass prism 
for SPR detection. Real-time diagnostics using an inexpensive disk and achieving high sensitivity have been performed by the fully automated system. We successfully provide an easy procedure to detect with one click three viruses within an hour.

\section{Conflict of Interests}

The authors declare that there is no conflict of interests regarding the publication of this paper.

\section{Acknowledgments}

This research was supported by the National Science Council of Taiwan under Project nos. NSC-98-2218-E-002-003 and NSC98-2622-E-002-003-CC1. Special thanks are due to Dr. Tsung-Liang Chuang and Dr. Nan-Fu Chiu.

\section{References}

[1] T. Iwamoto, T. Sonobe, and K. Hayashi, "Loop-mediated isothermal amplification for direct detection of Mycobacterium tuberculosis complex, M. avium, and M. intracellulare in sputum samples," Journal of Clinical Microbiology, vol. 41, no. 6, pp. 2616-2622, 2003.

[2] K. G. Beavis, M. B. Lichty, D. L. Jungkind, and O. Giger, "Evaluation of Amplicor PCR for direct detection of Mycobacterium tuberculosis from sputum specimens," Journal of Clinical Microbiology, vol. 33, no. 10, pp. 2582-2586, 1995.

[3] J. A. Down, M. A. O'Connell, M. S. Dey et al., "Detection of Mycobacterium tuberculosis in respiratory specimens by strand displacement amplification of DNA," Journal of Clinical Microbiology, vol. 34, no. 4, pp. 860-865, 1996.

[4] J. Lachnik, B. Ackermann, A. Bohrssen et al., "Rapid-cycle PCR and fluorimetry for detection of mycobacteria," Journal of Clinical Microbiology, vol. 40, no. 9, pp. 3364-3373, 2002.

[5] H. T. C. Thai, M. Q. Le, C. D. Vuong et al., "Development and evaluation of a novel loop-mediated isothermal amplification method for rapid detection of severe acute respiratory syndrome coronavirus," Journal of Clinical Microbiology, vol. 42, no. 5, pp. 1956-1961, 2004.

[6] M. Ito, M. Watanabe, N. Nakagawa, T. Ihara, and Y. Okuno, "Rapid detection and typing of influenza $\mathrm{A}$ and $\mathrm{B}$ by loop-mediated isothermal amplification: comparison with immunochromatography and virus isolation," Journal of Virological Methods, vol. 135, no. 2, pp. 272-275, 2006.

[7] S. Jayawardena, C. Y. Cheung, I. Barr et al., "Loop-mediated isothermal amplification for influenza A (H5N1) virus," Emerging Infectious Diseases, vol. 13, no. 6, pp. 899-901, 2007.

[8] L. L. M. Poon, B. W. Y. Wong, K. H. Chan et al., "Evaluation of real-time reverse transcriptase PCR and real-time loopmediated amplification assays for severe acute respiratory syndrome coronavirus detection," Journal of Clinical Microbiology, vol. 43, no. 7, pp. 3457-3459, 2005.

[9] M. Imai, A. Ninomiya, H. Minekawa et al., "Development of H5-RT-LAMP (loop-mediated isothermal amplification) system for rapid diagnosis of $\mathrm{H} 5$ avian influenza virus infection," Vaccine, vol. 24, no. 44, pp. 6679-6682, 2006.
[10] S. Iwata, Y. Shibata, J.-I. Kawada et al., "Rapid detection of Epstein-Barr virus DNA by loop-mediated isothermal amplification method," Journal of Clinical Virology, vol. 37, no. 2, pp. 128-133, 2006.

[11] H. Kaneko, T. Iida, K. Aoki, S. Ohno, and T. Suzutani, "Sensitive and rapid detection of herpes simplex virus and varicella-zoster virus DNA by loop-mediated isothermal amplification," Journal of Clinical Microbiology, vol. 43, no. 7, pp. 3290-3296, 2005.

[12] D. H. Paris, M. Imwong, A. M. Faiz et al., "Loop-mediated isothermal PCR (LAMP) for the diagnosis of falciparum malaria," The American Journal of Tropical Medicine and Hygiene, vol. 77, no. 5, pp. 972-976, 2007.

[13] L. L. M. Poon, B. W. Y. Wong, E. H. T. Ma et al., "Sensitive and inexpensive molecular test for falciparum malaria: defecting Plasmodium falciparum DNA directly from heat-treated blood by loop-mediated isothermal amplification," Clinical Chemistry, vol. 52, no. 2, pp. 303-306, 2006.

[14] M. Hagiwara, H. Sasaki, K. Matsuo, M. Honda, M. Kawase, and H. Nakagawa, "Loop-mediated isothermal amplification method for detection of human papillomavirus type 6, 11, 16, and 18," Journal of Medical Virology, vol. 79, no. 5, pp. 605-615, 2007.

[15] J. D. Scott and D. R. Gretch, "Molecular diagnostics of hepatitis $\mathrm{C}$ virus infection: a systematic review," The Journal of the American Medical Association, vol. 297, no. 7, pp. 724-732, 2007.

[16] C. M. Fan, Rapid detection of hepatitis $C$ virus by loop-mediated isothermal amplification [M.S. thesis], Department of Clinical Laboratory Sciences and Medical Biotechnology, National Taiwan University, 2009.

[17] P. Pancholi, F. Wu, and P. Della-Latta, "Rapid detection of cytomegalovirus infection in transplant patients," Expert Review of Molecular Diagnostics, vol. 4, no. 2, pp. 231-242, 2004.

[18] R. Gorkin, J. Park, J. Siegrist et al., "Centrifugal microfluidics for biomedical applications," Lab on a Chip, vol. 10, no. 14, pp. 1758-1773, 2010.

[19] M. Arens, "Clinically relevant sequence-based genotyping of HBV, HCV, CMV, and HIV," Journal of Clinical Microbiology, vol. 22, no. 1, pp. 11-29, 2001.

[20] C. T. Schembri, T. L. Burd, A. R. Kopf-Sill, L. R. Shea, and B. Braynin, "Centrifugation and capillarity integrated into a multiple analyte whole blood analyser," Journal of Automatic Chemistry, vol. 17, no. 3, pp. 99-104, 1995.

[21] M. J. Madou, L. J. Lee, S. Daunert, S. Lai, and C.-H. Shih, "Design and fabrication of CD-like microfluidic platforms for diagnostic: Mircofluidic functions," Biomedical Microdevices, vol. 3, no. 3, pp. 245-254, 2001.

[22] B. S. Lee, J.-N. Lee, J.-M. Park et al., "A fully automated immunoassay from whole blood on a disc," Lab on a ChipMiniaturisation for Chemistry and Biology, vol. 9, no. 11, pp. 1548-1555, 2009.

[23] C. N. Lee and C. W. Lin, "Clinical validation of soft materialbased OLED SPR biosensor," Project Report No. NSC 96-2218E-002-026, National Science Council, Taipei, Taiwan, 2008.

[24] T. Cai, G. Lou, J. Yang, D. Xu, and Z. Meng, "Development and evaluation of real-time loop-mediated isothermal amplification for hepatitis B virus DNA quantification: a new tool for HBV management," Journal of Clinical Virology, vol. 41, no. 4, pp. 270-276, 2008.

[25] Q. Li, X. Ma, R. Zhou et al., "Detection of HCV gene by reverse transcription loop-mediated isothermal amplification method," Chinese Journal of Virology, vol. 22, no. 5, pp. 334-338, 2006. 
[26] S.-Y. Lee, C.-N. Lee, H. Mark, D. R. Meldrum, and C.-W. Lin, "Efficient, specific, compact hepatitis B diagnostic device: optical detection of the hepatitis B virus by isothermal amplification," Sensors and Actuators, B: Chemical, vol. 127, no. 2, pp. 598-605, 2007.

[27] T. Brenner, T. Glatzel, R. Zengerle, and J. Ducrée, "Frequencydependent transversal flow control in centrifugal microfluidics," Lab on a Chip, vol. 5, no. 2, pp. 146-150, 2005.

[28] J. M. Chen, P.-C. Huang, and M.-G. Lin, "Analysis and experiment of capillary valves for microfluidics on a rotating disk," Microfluidics and Nanofluidics, vol. 4, no. 5, pp. 427-437, 2008.

[29] K.-C. Liu, Y.-H. Lu, Y.-H. Liao, and B.-S. Huang, "Utilizing transparent $\mathrm{ZnO}$ thin film as permeation barrier to improve light outcoupling and longevity of top-emission polymer lightemitting devices," Japanese Journal of Applied Physics, vol. 47, no. 4, pp. 3162-3166, 2008. 

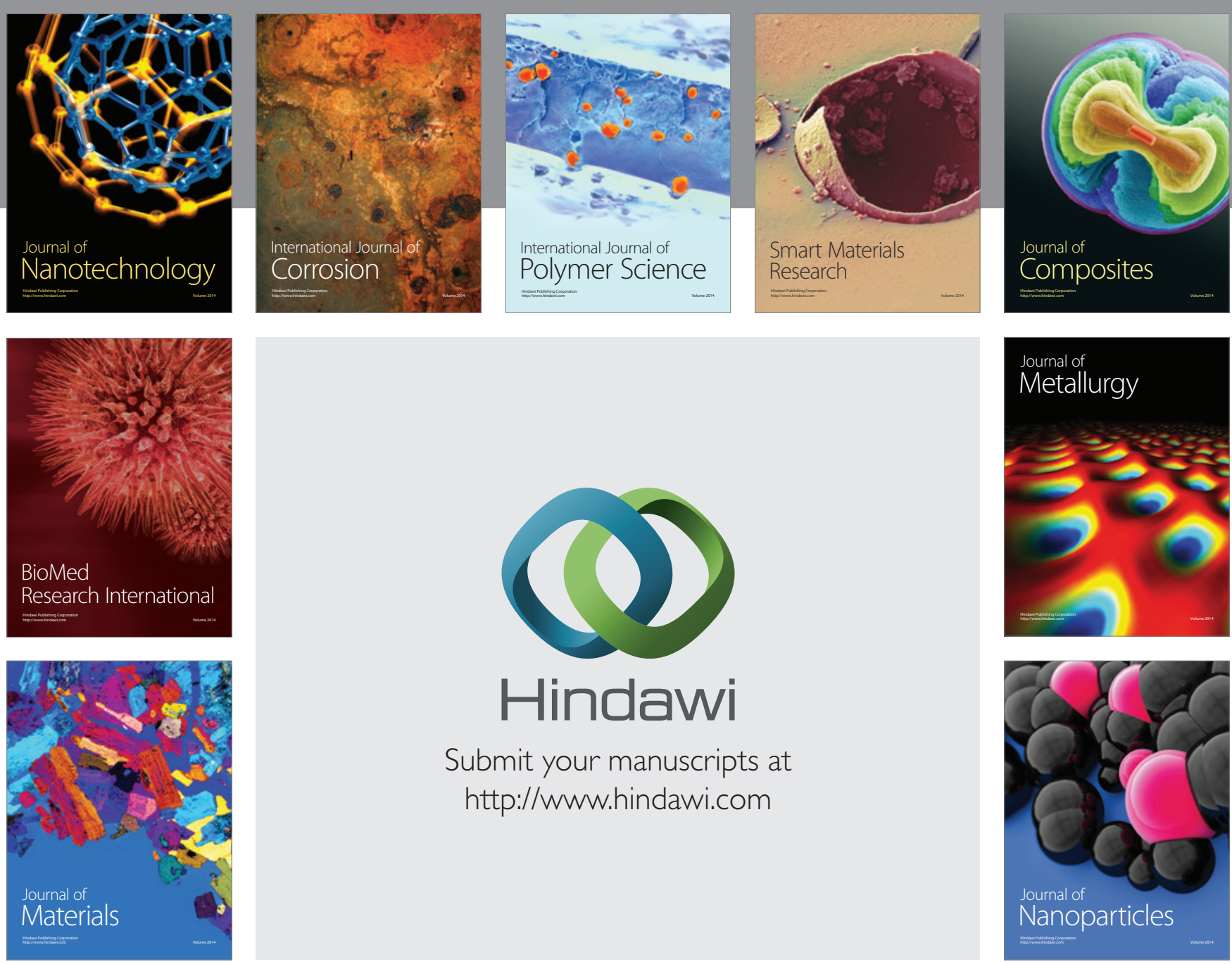

Submit your manuscripts at http://www.hindawi.com
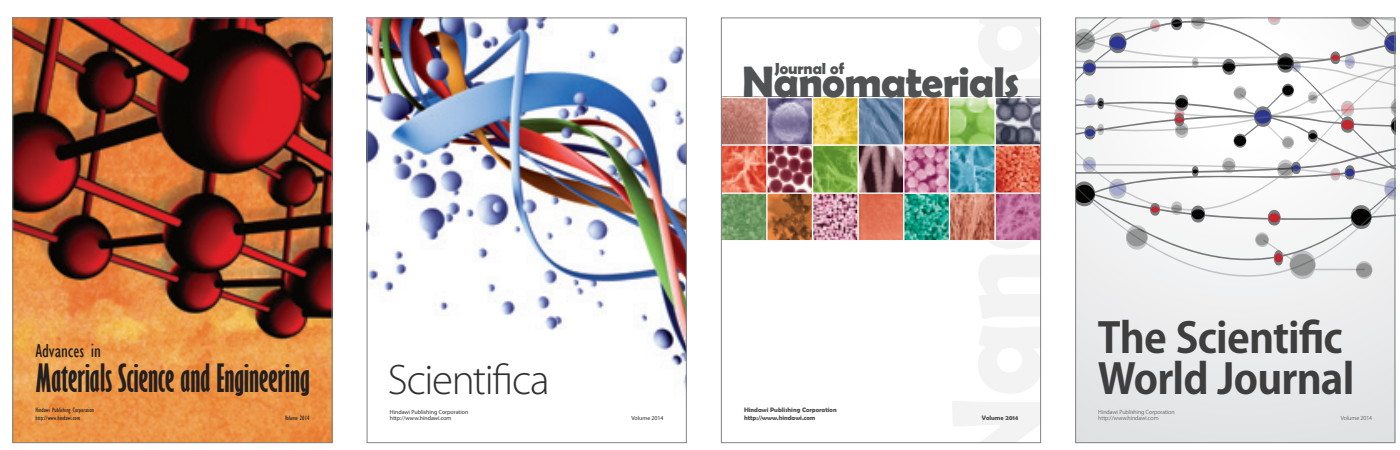

\section{The Scientific World Journal}
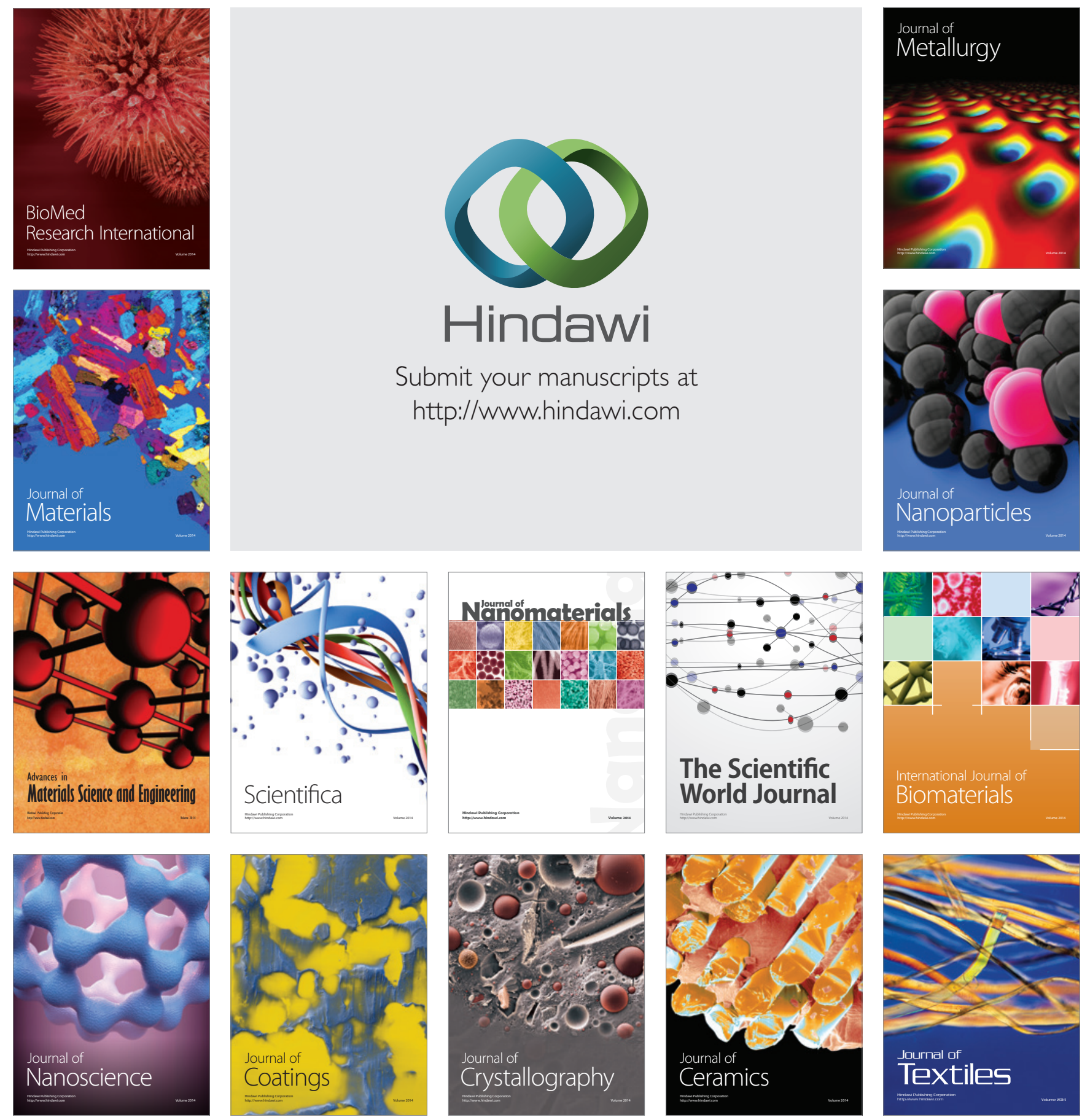